Cahiers $d u$ MONDE RUSSE

\section{Cahiers du monde russe}

Russie - Empire russe - Union soviétique et États indépendants

$46 / 4 \mid 2005$

L'invention d'une politique humanitaire

\title{
A. G. Man'kov et al., éds., Rossijskoe gosudarstvo v XIV-XVII vV.
}

André Berelowitch

\section{OpenEdition}

\section{Journals}

Édition électronique

URL : https://journals.openedition.org/monderusse/2886

DOI : 10.4000/monderusse.2886

ISSN : $1777-5388$

Éditeur

Éditions de l'EHESS

Édition imprimée

Date de publication : 1 décembre 2005

Pagination : 859-862

ISBN : 2-7132-2057-2

ISSN : $1252-6576$

Référence électronique

André Berelowitch, «A. G. Man'kov et al., éds., Rossijskoe gosudarstvo v XIV-XVII vv. », Cahiers du monde russe [En ligne], 46/4 | 2005, mis en ligne le 29 juin 2009, consulté le 04 septembre 2022. URL http://journals.openedition.org/monderusse/2886 ; DOI : https://doi.org/10.4000/monderusse.2886

Ce document a été généré automatiquement le 4 septembre 2022

Tous droits réservés 


\title{
A. G. Man'kov et al., éds., Rossijskoe gosudarstvo v XIV-XVII vv.
}

\author{
André Berelowitch
}

\section{RÉFÉRENCE}

A. G. MAN'KOV, Rossijskoe gosudarstvo v XIV-XVII vv. Sbornik statej, posvjaščennyj 75-letiju so dnja roždenija Ju. G. Alekseeva [L'État russe, XIV ${ }^{\mathrm{e}}$ XVII ${ }^{\mathrm{e}}$ siècles. Recueil d'articles consacré au $75^{\mathrm{e}}$ anniversaire de Ju. G Alekseev] SaintPétersbourg : Dmitrij Bulanin, 2002, 616 p.

1 Tout volume de mélanges nous renseigne à la fois sur son destinataire, sur les sujets préférés des historiens, et bien sûr sur ces sujets eux-mêmes. Le recueil offert à Jurij Georgievič Alekseev ne fait pas exception. Jurij Alekseev, dont l'ouvrage sur le district de Perejaslavl'-Zalesskij fit sensation en 1966, appartient à la seconde génération de spécialistes de l'histoire agraire, celle qui succéda aux pionniers, Boris D. Grekov, Ivan I. Smirnov, Stepan B. Veselovskij. Il n'était pas le premier à rédiger une monographie régionale (Aleksandr Kopanev l'avait précédé, avec une étude sur la possession foncière de Beloozero), mais personne avant lui n'avait entrepris de retracer l'évolution de la propriété foncière à travers l'histoire des principaux acteurs: paysannerie, clergé et noblesse $e^{1}$. Depuis, il a continué à s'intéresser à l'histoire sociale, mais en se tournant de plus en plus vers celle du droit et, plus récemment, vers l'histoire politique $\mathrm{du} \mathrm{Xv}^{\mathrm{e}}$ siècle.

2 La composition du recueil privilégie, comme il se doit, les centres d'intérêt de Jurij Alekseev, mais tient compte également des tendances récentes. Deux articles seulement traitent d'histoire économique, devenue la parente pauvre de l'historiographie russe. Anna L. Horoškevič revient au glossaire allemand-russe de Tönnies Fonne (1607), cette fois pour éclairer les opérations de crédit entre les marchands de la Hanse et ceux de Pskov. A. I. Razdorskij étudie le marché de Možajsk à partir de registres d'octroi qui ne couvrent que sept ans sur une période de trente (1644-1645 à 1673-1674). S'ils suffisent à mettre en évidence le mouvement saisonnier des affaires (les deux tiers des ventes ont 
lieu en hiver), ils ne lui permettent pas de tirer de conclusions sur l'évolution globale du commerce local au cours de la période. En revanche, l'histoire de l'Église, celle de la noblesse et les études prosopographiques en général tiennent une place importante. $\mathrm{Ne}$ seront présentées ici que celles des contributions qui peuvent intéresser un public assez large ; on laissera de côté, quel que soit leur mérite, celles qui se confinent dans le cercle strict de leur spécialité.

3 Vasilij F. Andreev consacre quelques pages bien venues à un vieux problème: de quand datent les « cinquièmes » (pjatiny) entre lesquels était partagé le territoire de Novgorod? Sans apporter, il le souligne lui-même, de preuve décisive, il incline à penser qu'ils sont antérieurs à l'annexion par Moscou, et que chacun était administré par l'un des cinq quartiers (koncy) de la ville. La localisation des terres monastiques et le contenu des lettres sur écorce de bouleau lui permettent d'esquisser, avec quelques incertitudes, une correspondance entre quartiers et cinquièmes, différente de celle proposée naguère par George Vernadsky². Mihail M. Krom se penche, après bien d'autres, sur la question des effectifs de l'armée russe au Xvi ${ }^{e}$ siècle, dont les estimations varient, selon les historiens, entre 50000 et 150000 combattants. Il établit une corrélation ingénieuse entre les chiffres avancés par les chroniques de toutes origines et le nombre des capitaines (voevody) qui figure dans les registres des rangs (razrjadnye knigi), ce qui l'amène à un effectif mobilisable de plus de 100000 soldats. Mais il omet de nous dire comment, même en tenant compte des provisions que les militaires étaient tenus d'emporter en campagne, une telle foule pouvait vivre sur le pays - ce qui affaiblit quelque peu la portée de sa démonstration. Une série de documents nouveaux, dont la déposition du lieutenant-colonel de mousquetaires Lužin (publiée en annexe) qui contredit sur certains points essentiels le journal de Patrick Gordon ${ }^{3}$, amène Pavel V. Sedov à revoir entièrement le récit de la défense de Čigirin contre les Turcs en 1677 et à nuancer les affirmations communément admises, d'une part sur l'impréparation des troupes russes, notamment des régiments de nouvelle ordonnance, d'autre part sur les tensions entre Russes et Ukrainiens. Tat'jana I. Paškova montre comment la législation de la Russie ancienne tend d'abord à généraliser et allonger les peines de prison, puis évolue en sens inverse, à cause du surpeuplement des locaux de détention. Surtout, elle met en évidence le changement d'attitude de la monarchie qui, à partir du milieu du XVII siècle, renonce progressivement au vieux système qui confiait la police, la justice et la répression à des officiers élus par la population pour intervenir elle-même. Sans apporter d'éléments absolument nouveaux, mais avec cette vraisemblance psychologique dont il a le secret, Ruslan G. Skrynnikov reconstitue, en la confrontant au drame de Puškin, la véritable personnalité et la carrière politique de Boris Godunov.

4 D'autres historiens soumettent des sources inédites à l'attention des chercheurs. C'est ainsi que Marija V. Korogodina s'interroge sur l'apparition dans les manuels de confession, à la fin $d u x^{e}$ et au début $d u X V I^{e}$ siècle, de questions visant les péchés des grands (trahison, corruption, abus de pouvoir, mauvais traitement des serviteurs). Certaines fautes sont sanctionnées par le Justicier de 1497, ce qui pourrait expliquer la date de ces innovations, mais la plupart ne constituent pas des délits. Le but de l'Église russe est donc bien d'inculquer aux fidèles une morale plus exigeante. Anastasija A. Romanova, qui a publié un ouvrage érudit sur la chronologie et le comput ecclésiastique dans l'ancienne Russie $e^{4}$ s'intéresse ici aux marginalia des tables pascales et aux spéculations qu'elles révèlent sur la fin du monde, attendue par certains en l'an 7000 de l'ère byzantine, c'est-à-dire en 1492. Jurij M. Eskin a trouvé dans une affaire de préséance 
de 1642, qu'il publie intégralement ${ }^{5}$, des informations sur le soulèvement de la ville de Tihvin, en 1613. Parmi les cinq articles consacrés aux monastères, on retiendra celui de Marina S.Čerkasova, grande spécialiste de la Trinité-Saint-Serge, sur le personnel des chancelleries monastiques et la liste des acquisitions foncières de Saint-Cyrille de Beloozero entre 1601 et 1700, établie par Aleksandr Gorfunkel', mais revue et éditée par Zoja V. Dmitrieva, qui publie par ailleurs des chartes statutaires du même couvent.

Enfin, quatre articles importants d'histoire sociale ouvrent la voie à de nouvelles recherches. On connaît mal le fonctionnement des communautés paysannes en pays de seigneurie, entre autres à cause du manque d'archives. Celles du monastère de NotreDame d'Ibérie, étudiées par Zoja A. Timošenkova, révèlent une autonomie comparable à celle de la paysannerie libre du Nord. Les serfs du couvent, réunis plusieurs fois par an en assemblée plénière, élisent leurs syndics (starosty); ceux-ci ont assez de compétence, d'autorité et de moyens pour envoyer leurs émissaires à Moscou solliciter le retour des terres monastiques aux domaines de la couronne.

6 Konstantin V. Petrov fait le point sur le problème du prožitok, cette portion du domaine en précaire, ou pomest'e, accordée à la veuve et aux enfants des nobles à la mort du chef de famille. Bien informé des travaux publiés hors de Russie, servi par sa méthode pointilleuse d'historien du droit, il montre comment l'évolution spontanée de la jurisprudence (car la monarchie n'essaie pas de légiférer avant la première moitié du XVII siècle) conduit à un résultat paradoxal : la veuve peut garder le prožitok même si elle se remarie, mais non le patrimoine héréditaire, qui retourne au lignage de son défunt époux.

7 Andrej P. Pavlov, qui s'est fait connaître par un ouvrage sur la cour au temps de Boris Godunov $^{6}$, a étendu ses recherches à la noblesse du XVII ${ }^{e}$ siècle. Son analyse des pages (žil 'cy), qui sont passés en revue en 1643, est un modèle du genre, parce qu'elle combine les données prosopographiques (liste alphabétique des 1222 pages, avec leur âge, leur ancienneté, leurs possessions foncières) avec l'histoire institutionnelle: les pages, au terme d'un stage à la cour qui peut aller jusqu'à vingt ans, sont promus dans les grades de Moscou ou, s'ils ne se sont distingués en rien, pas même par leur lignage, versés dans la noblesse de province.

8 Vladimir A. Arakčeev, qui se spécialise en histoire agraire, s'est attaqué à un texte législatif important, l'établissement du 9 mars 1607, qui prolonge jusqu'à quinze ans le délai de prescription en matière de poursuite des serfs fugitifs et constitue donc, s'il est authentique, un jalon crucial dans la genèse du servage. L'auteur démontre, de manière convaincante me semble-t-il, que le texte est doublement anachronique, par son contenu et par son vocabulaire. Un édit de 1597 fixait le délai de prescription à cinq ans, et il faut attendre 1641 pour qu'il passe à quinze ans : comment expliquer le bond en avant de 1607, suivi d'un retour en arrière? Par ailleurs, les termes employés sont caractéristiques non pas du début, mais de la fin du XVII e siècle. Ces anomalies s'expliquent lorsque l'on sait que l'établissement nous est parvenu à travers une copie de Vasilij N. Tatiščev (1686-1750) : l'historien du XVIII siècle a probablement essayé de reconstituer, selon ses lumières, un texte dont il n'avait retrouvé que des fragments.

-Ju. G. Alekseev, Agrarnaja i social'naja istorija Severo-Vostočnoj Rusi XV-XVI vv. Perejaslavskij uezd, M.-L., 1966, 268 p. 


\begin{tabular}{||l|l|}
\hline Quartiers & Cinquièmes \\
\hline Nerevskij & Vodskaja \\
\hline Ljudin & Šelonskaja \\
\hline Slavenskij & Derevskaja \\
\hline Plotnickij & Obonežskaja \\
\hline Zagorodskij & Bežeckaja \\
\hline
\end{tabular}

- Une traduction russe, par Dmitrij G. Fedosov, est en cours de parution : Patrik Gordon, Dnevnik 1635-1659, M. : Nauka, 2001 ; 1659-1667, 2002 ; 1677-1678, 2005.

-A. A. Romanova, Drevnerusskie kalendarno-hronologičeskie istočniki XV-XVII vv., SPb. : Dmitrij Bulanin, 2002, $432 \mathrm{p}$.

- $\mathrm{N}^{\circ} 1399$ dans son répertoire, Ju. M. Eskin, Mestničestvo v Rossii XVI-XVII vv. Hronologičeskij reestr, M. : Arheografičeskij Centr, 1994.

-A. P. Pavlov, Gosudarev dvor i političeskaja bor'ba pri Borise Godunove (1584-1605 gg.), SPb. : Nauka, 1992, 280 p. 